\title{
Enhanced Soft-Handoff Scheme for Real-Time Streaming Services in Intelligent Transportation Systems Based on CDMA
}

\author{
Young-uk Chung and Dong-Ho Cho, Senior Member, IEEE
}

\begin{abstract}
Code division multiple access (CDMA)-based radioon-fiber road-vehicle communication systems were proposed to support real-time streaming services in intelligent transportation systems (ITSs). Soft handoff should be provided for real-time streaming services because these services are required to guarantee real-time property and quality of service $(\mathrm{Q} o \mathrm{~S})$. However, soft handoff of real-time streaming services causes the shortage of resource in view of the system. In this paper, the doubleadjustment soft-handoff (DASH) scheme is proposed to support seamless service regardless of the shortage of resource and maximize the resource efficiency when real-time streaming services are served in CDMA-based ITSs. The DASH scheme adjusts the data rate of a call when it starts and finishes soft handoff. The performance of the DASH scheme is evaluated by analytical and simulation methods. Performances are evaluated with respect to the blocking probability, the handoff failure probability, and the carried traffic. Numerical results show that the proposed scheme can give relative good maintenance of calls and good throughput.
\end{abstract}

Index Terms-Real-time multimedia service, soft handoff, streaming service.

\section{INTRODUCTION}

$\mathbf{R}$ APIDLY increasing demands for intelligent transportation systems (ITSs) have been required to support various multimedia services. There have been researches and developments about several services such as electronic toll collection, vehicle location, and navigation services [1], [2]. In the near future, real-time streaming services that require high data rate become core applications. These real-time streaming services require broadband wireless communication under highmobility conditions.

There remain many problems such as signal distortion and information loss to support broadband wireless communication in ITS. To solve these problems, code division multiple access (CDMA)-based radio-on-fiber ( $\mathrm{ROF}$ ) road-vehicle communication systems were proposed [1]. The use of the ROF technique makes the construction of cost-effective micro-

Manuscript received September 21, 2004; revised June 3, 2005. This work was supported by a research grant from Kwangwoon University in 2006. The Associate Editor for this paper was R. Kohno.

Y. Chung is with the Department of Electronic Engineering, Kwangwoon University, Seoul, Korea (e-mail: yuchung@kw.ac.kr).

D.-H. Cho is with the Department of Electrical Engineering and Computer Science, Korea Advanced Institute of Science and Technology (KAIST), Daejeon, Korea (e-mail: dhcho@ee.kaist.ac.kr).

Digital Object Identifier 10.1109/TITS.2006.874715 cell systems, which are requisite for high-capacity multimedia services. CDMA is one of the most promising technologies for mobile communication systems. The employment of CDMA enables this system to offer various unique features such as robustness against fast fading by the use of diversity reception, equalization, interleaving, RAKE receivers, and soft handoff.

There are three kinds of resource in the CDMA-based ITS: orthogonal code, physical channel card (modem), and transmission power. The hard capacity of the system is affected by the orthogonal code and the physical channel card, and soft capacity is affected by the transmission power. Soft handoff is a technique that makes mobile stations (MSs) residing in an overlapping cell region communicate with both the currently serving base station (BS) and the target BS, simultaneously. Due to perfect frequency reuse feature of CDMA, MSs can perform soft handoff when they move between cells. It is commonly known that soft handoff consumes more resource than hard handoff on the forward link with respect to hard capacity, even though it can support reliable service, better communication quality due to double usage of hard capacity, and interference reduction [3]-[6]. On the other hand, the waste of soft capacity on the forward link can be compensated by the macrodiversity.

In streaming services, which we consider in ITS, soft handoff should be provided because these services require the guarantee of real-time property and quality of service (QoS). However, there remains a serious problem when we apply soft handoff to these services, because these services require multiple orthogonal code and physical channel card simultaneously to support high-speed transmissions. For example, when an MS that requires four codes and channel cards is in the state of soft handoff, it consumes eight codes and channel cards for the system's point of view. It causes the shortage of system capacity such as frequent handoff-call dropping. Because seamlessness is very important feature for real-time services, this problem should be overcome.

Considering the shortage of hard capacity, there have been many previous works on resource management for real-time streaming services. However, most of these previous works focused on access control for integrated voice/data/video traffics [7]-[14]. Only few researches have been focused on resource allocation during soft handoff of real-time streaming services. In this paper, we propose the double-adjustment softhandoff (DASH) scheme to compensate for the shortage of hard 
capacity and maximize the resource efficiency. The proposed DASH scheme adjusts the data rate of a call when the call starts and finishes soft handoff, respectively.

This paper is organized as follows. In Section II, we explain the proposed soft-handoff scheme to maximize the resource efficiency. In Section III, we describe the system model, develop the analytic model, and define performance measures to evaluate the proposed scheme. In Section IV, we describe the simulation environments, perform numerical results, and compare the results. Finally, we summarize our results and conclude this paper in Section V.

\section{DASH SCHEME}

In this section, we describe the algorithm of our proposed DASH scheme. The system considered in this paper is an allIP network-based ITS system. This system consists of an IPbased core network and a CDMA-based radio access network. The radio access network of this system consists of microcells that have small range. In the ITS, various real-time streaming services are provided to fast-moving vehicles. Because realtime streaming services have a long service time, an MS passes through many cells during the call duration. Therefore, an efficient resource allocation during soft handoff is necessary to support seamless service.

When an MS that supports real-time streaming service is in the state of soft handoff, the same hard capacity is required for both the serving BS and the target BS. Because real-time streaming services require a large amount of resource, handoffcall dropping occurs frequently. The shortage of hard capacity such as the orthogonal code and the channel card becomes a severe problem in view of the system. The DASH scheme is proposed to alleviate this problem by adjusting the data rate of an MS during soft handoff. We assume that the data rate of an MS is determined by only the amount of occupied resource, which consists of the orthogonal code and the physical channel card.

The algorithm of the DASH scheme is shown in Fig. 1. When a new call is originated in the normal region, it requests resource to the serving BS based on its service type. If there remains enough resource, the requested resource is allocated to the call. Otherwise, the call is blocked.

When an on-going call in the normal region moves into the handoff region, the call sends a negotiate message to the target cell BS and adjusts its current data rate according to the remaining resource in the target cell BS. If the target BS has enough remaining resource, the adjustment is accepted and the call is in the soft-handoff state. Otherwise, the call is dropped.

When a call in the soft-handoff state moves out of the handoff region and enters into the normal region of the target cell, the call breaks communication with its serving cell BS, sends a negotiate message to the target cell BS, and adjusts its current data rate according to the remaining resource in both the serving BS and the target BS. If both BSs have enough remaining resource, the adjustment is accepted and the adjusted resource is allocated to the call.

When a new call is generated in the handoff region, it requests resource to BSs, which constitute the handoff region according to its data rate. If there is sufficient available resource in both BSs, the call is in soft-handoff state and communicates with both BSs. If there is no sufficient resource in at least one of both BSs, the call is blocked.

\section{Performance Analysis}

In this section, we analyze the performance of the proposed DASH scheme. It alleviates a shortage of hard capacity by adjusting the data rate of MSs during soft handoff. This section consists of three parts. In the first part, we describe the system model of our analysis. In the second part, we perform a mathematical analysis to calculate performance considering hard capacity. The analysis is performed using the Markov chain method. We referred to the approach that was used by Chung and Cho [15]. The performance measure is defined in the third part. Performance is evaluated with respect to the blocking probability, the handoff failure probability, and the carried traffic.

\section{A. System Modeling}

The system we consider in this paper is the road-vehicle ITS. In the road-vehicle ITS, all microcells are located along the streets. Therefore, all MSs move in just two directions. We assume that there is no turning back. The system description is shown in Fig. 2. The target service is real-time streaming service such as video on demand ( $\mathrm{VoD})$ and videophone. In this system model, we use the term "resource" as a minimum unit of capacity. The minimum requirement for maintaining a call is one orthogonal code and one physical channel card. Thus, the term "resource" can imply either the orthogonal code or the physical channel card. We assume that there are $N$ kinds of service types. Each service type needs resource ranging from one resource to $N$ resource according to its property. The more an MS uses resource, the higher the data rate the MS uses.

We divide a cell into two regions such as the normal region and the handoff region, as shown in Fig. 2. The normal region is surrounded by the handoff region. Let the ratio of the handoff region in a cell be $f$. Then, $f$ is given by

$$
f=\frac{\text { area of handoff region }}{\text { area of a cell }} .
$$

We assume that the handoff region is made by only two cells. Let the handoff-call-arrival rate be $\lambda_{\mathrm{h}}$. The new call-arrival rate is assumed to be Poisson distribution with rates $\lambda_{\mathrm{n}}$. Let the new call-arrival rates in the normal region and the handoff region be $\lambda_{\text {nn }}$ and $\lambda_{\text {nh }}$, respectively. Under the assumption that new call arrivals are uniformly distributed, $\lambda_{\mathrm{nn}}$ and $\lambda_{\mathrm{nh}}$ are found to be

$$
\begin{aligned}
\lambda_{\mathrm{nn}} & =(1-f) \cdot \lambda_{\mathrm{n}} \\
\lambda_{\mathrm{nh}} & =f \cdot \lambda_{\mathrm{n}} .
\end{aligned}
$$

We assume that every handoff requirement can be perfectly detected in our model and the resource assignment is made instantaneously if resource is available. The total allowable resource of a cell is assumed to be $C$.

Let the call-duration time be $T_{\mathrm{C}}$. We assume that $T_{\mathrm{C}}$ is exponentially distributed with mean $\mu_{\mathrm{C}}^{-1}$. Let the mean dwell time in the whole cell, the mean dwell time in the normal 


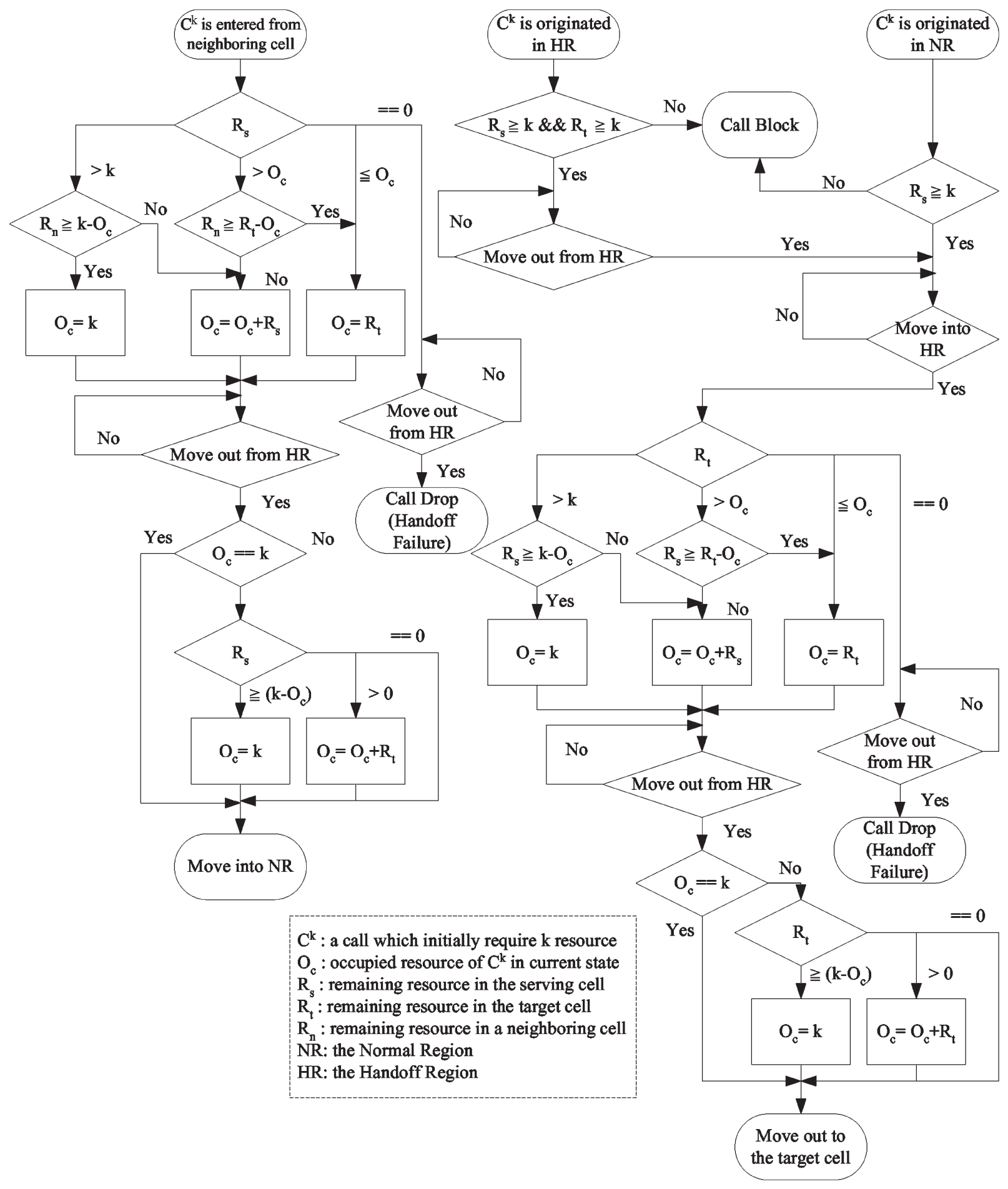

Fig. 1. Algorithm of the DASH scheme.

region, and the mean dwell time in the handoff region be $T_{\mathrm{dg}}$, $T_{\mathrm{dn}}$, and $T_{\mathrm{dh}}$, respectively. $T_{\mathrm{dg}}, T_{\mathrm{dn}}$, and $T_{\mathrm{dh}}$ are also assumed to be exponentially distributed with mean $\mu_{\mathrm{dg}}^{-1}, \mu_{\mathrm{th}}^{-1}$, and $\mu_{\mathrm{tn}}^{-1}$, respectively. There are relations between the mean dwell time in a cell and the mean dwell time in each region. Because the target system of this paper is ITS, the related functions are described as follows:

$$
\begin{aligned}
& T_{\mathrm{dn}}=(1-f) \cdot T_{\mathrm{dg}} \\
& T_{\mathrm{dh}}=f \cdot T_{\mathrm{dg}} .
\end{aligned}
$$

\section{B. System Analysis}

The state of a cell $s$ is defined as

$$
\left\{\begin{array}{cccc}
c_{1}(s) & c_{2}(s) & \cdots & c_{N}(s) \\
c_{1}^{\text {hi }}(s) & c_{2}^{\text {hi }}(s) & \cdots & c_{N}^{\text {hi }}(s) \\
c_{1}^{\text {ho }}(s) & c_{2}^{\text {ho }}(s) & \cdots & c_{N}^{\text {ho }}(s)
\end{array}\right\}
$$

where $c_{k}(s)$ is the number of calls in the normal region occupying $k$ resource when the cell is in state $s, c_{k}^{\mathrm{hi}}(s)$ is the number of calls in the handoff region, which are moved from 


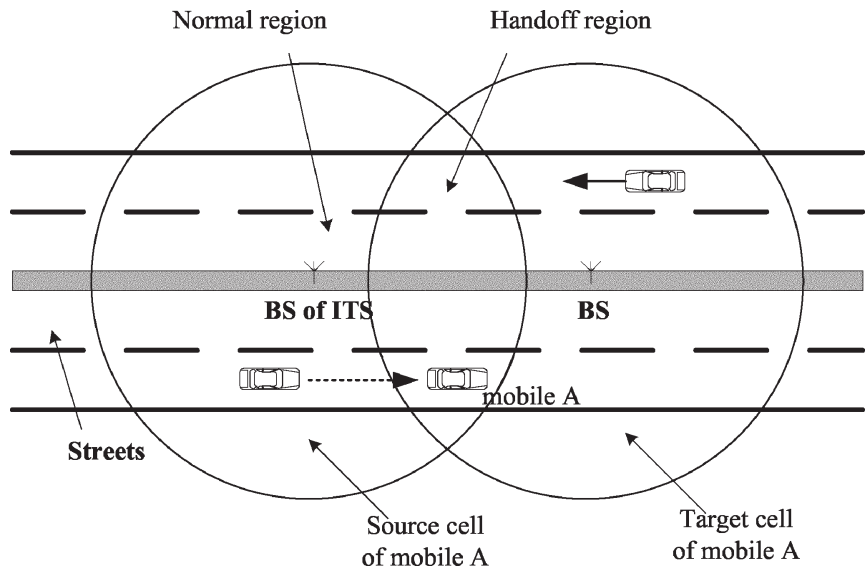

Fig. 2. System description.

the neighbor cell with occupying $k$ resource when the current cell is in state $s$, and $c_{k}^{\text {ho }}(s)$ is the number of calls in the handoff region, which are moved from the normal region of the current cell with occupying $k$ resource when the cell is in state $s$. The state $s$ has a value ranging from 0 to $s_{\max } \cdot c_{k}(s), c_{k}^{\text {hi }}(s)$, and $c_{k}^{\text {ho }}(s)$ can have a value ranging from 0 to $\lfloor C / k\rfloor$.

Let $R_{\mathrm{n}}(s)$ be the number of resource occupied by calls in the normal region when the cell is in state $s$, and $R_{\mathrm{h}}(s)$ be the number of resource occupied by calls in the handoff region when the cell is in state $s$. Then, $R_{\mathrm{n}}(s)$ and $R_{\mathrm{h}}(s)$ are calculated as

$$
\begin{aligned}
& R_{\mathrm{n}}(s)=\sum_{i=1}^{N} i \cdot c_{i}(s) \\
& R_{\mathrm{h}}(s)=\sum_{i=1}^{N}\left(i \cdot c_{i}^{\mathrm{hi}}(s)+i \cdot c_{i}^{\mathrm{ho}}(s)\right) .
\end{aligned}
$$

$R_{\mathrm{n}}(s)+R_{\mathrm{h}}(s)$ should not be bigger than total allowable resource $C$.

There are seven types of state transition. The first type is a state transition due to a new call arrival in the normal region. Let $r_{\mathrm{nn}}\left(x_{2}, x_{1}\right)$ be the flow component from state $x_{1}$ to state $x_{2}$ due to a new call arrival in the normal region. Also, let $\alpha_{k}$ be the probability that the new call belongs to a service type that needs $k$ resource. $\alpha_{k}$ has a predefined arbitrary value. As we assume that there are $N$ kinds of services, $k$ has the value ranging from 1 to $N$. According to the conventional soft-handoff strategy, if there remain $k$ resource in a cell and a new call requires $k+1$ resource, the new call is blocked. Therefore, if we define the state $x_{k}$ as

$\left\{\begin{array}{ccccc}c_{1}(s) & \cdots & \left(c_{k}(s)+1\right) & \cdots & c_{N}(s) \\ c_{1}^{\text {hi }}(s) & & \cdots & & c_{N}^{\text {hi }}(s) \\ c_{1}^{\text {ho }}(s) & & \cdots & & c_{N}^{\text {ho }}(s)\end{array}\right\}$

where $(1 \leq k \leq N)$

then $r_{\mathrm{nn}}\left(x_{k}, s\right)$ is expressed as

$$
r_{\mathrm{nn}}\left(x_{k}, s\right)=\left\{\begin{array}{ll}
\lambda_{\mathrm{nn}} \cdot \alpha_{k}, & \text { for } C-\left(R_{\mathrm{n}}(s)+R_{\mathrm{h}}(s)\right) \geq k \\
& \text { and }\left(c_{k}(s)+1\right) \cdot k \leq C \\
0, & \text { otherwise }
\end{array} .\right.
$$

The second type is a state transition due to a new call arrival in the handoff region. Let $r_{\mathrm{nh}}\left(x_{2}, x_{1}\right)$ be the flow component from state $x_{1}$ to state $x_{2}$ due to the new call arrival in the handoff region. If we define the state $x_{1}$ as

$$
\begin{aligned}
& \left\{\begin{array}{ccccc}
c_{1}(s) & & \cdots & & c_{N}(s) \\
c_{1}^{\text {hi }}(s) & \cdots & \left(c_{k}^{\text {hi }}(s)+1\right) & \cdots & c_{N}^{\text {hi }}(s) \\
c_{1}^{\text {ho }}(s) & & \cdots & & c_{N}^{\text {ho }}(s)
\end{array}\right\} \\
& \text { where }(1 \leq k \leq N)
\end{aligned}
$$

and the state $x_{2}$ as

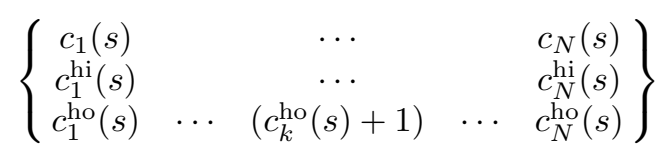

where $(1 \leq k \leq N)$

then $r_{\mathrm{nh}}\left(x_{1}, s\right)$ and $r_{\mathrm{nh}}\left(x_{2}, s\right)$ are expressed as

$$
\begin{aligned}
& r_{\mathrm{nh}}\left(x_{1}, s\right)=\left\{\begin{array}{ll}
\lambda_{\mathrm{nh}} \cdot \alpha_{k} / 2, & \text { for } C-R_{\mathrm{n}}(s)-R_{\mathrm{h}}(s) \geq k \\
\text { and }\left(c_{k}^{\mathrm{hi}}+1\right) \cdot k \leq C & \text { otherwise }
\end{array},\right. \\
& r_{\mathrm{nh}}\left(x_{2}, s\right)=\left\{\begin{array}{ll}
\lambda_{\mathrm{nh}} \cdot \alpha_{k} / 2, & \text { for } C-R_{\mathrm{n}}(s)-R_{\mathrm{h}}(s) \geq k \\
\text { and }\left(c_{k}^{\mathrm{ho}}+1\right) \cdot k \leq C & \text { otherwise }
\end{array} .\right.
\end{aligned}
$$

New calls in the handoff region are counted in $c_{k}^{\text {hi }}$ and $c_{k}^{\text {ho }}$ according to their moving direction. As we assumed that there can be only two directions in this system, the probabilities that a new call in the handoff region is counted in $c_{k}^{\text {hi }}$ and $c_{k}^{\text {ho }}$ are 0.5 , respectively. The term $1 / 2$ in (8) is included due to this assumption.

The third type is a state transition due to call completion. Let $r_{\mathrm{c}}\left(x_{2}, x_{1}\right)$ be the flow component from state $x_{1}$ to state $x_{2}$ due to call completion. If we define the state $x_{k}$ as

$\left\{\begin{array}{ccccc}c_{1}(s) & \cdots & \left(c_{k}(s)-1\right) & \cdots & c_{N}(s) \\ c_{1}^{\text {hi }}(s) & & \cdots & & c_{N}^{\text {hi }}(s) \\ c_{1}^{\text {ho }}(s) & & \cdots & & c_{N}^{\text {ho }}(s)\end{array}\right\}$

where $(1 \leq k \leq N)$

the state $x_{k}^{\text {hi }}$ as

$\left\{\begin{array}{ccccc}c_{1}(s) & & \cdots & & c_{N}(s) \\ c_{1}^{\mathrm{hi}}(s) & \cdots & \left(c_{k}^{\mathrm{hi}}(s)-1\right) & \cdots & c_{N}^{\mathrm{hi}}(s) \\ c_{1}^{\mathrm{ho}}(s) & & \cdots & & c_{N}^{\text {ho }}(s)\end{array}\right\}$ where $(1 \leq k \leq N)$

and the state $x_{k}^{\text {ho }}$ as

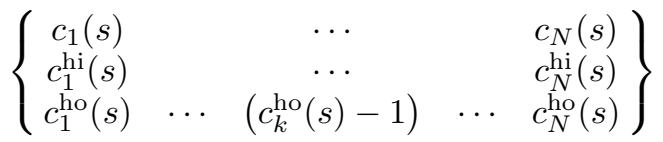

where $(1 \leq k \leq N)$ 
then $r_{\mathrm{c}}(x, s)$ is expressed as

$$
\begin{gathered}
r_{\mathrm{c}}\left(x_{k}, s\right)=k \cdot \mu \cdot c_{k}(s) \\
\quad \text { for } 1 \leq k \leq N \text { and } c_{k}(s)-1 \geq 0 \\
r_{\mathrm{c}}\left(x_{k}^{\mathrm{hi}}, s\right)=k \cdot \mu \cdot c_{k}^{\mathrm{hi}}(s) \\
\quad \text { for } 1 \leq k \leq N \text { and } c_{k}^{\mathrm{hi}}(s)-1 \geq 0 \\
r_{\mathrm{c}}\left(x_{k}^{\mathrm{ho}}, s\right)=k \cdot \mu \cdot c_{k}^{\mathrm{ho}}(s) \\
\quad \text { for } 1 \leq k \leq N \text { and } c_{k}^{\mathrm{ho}}(s)-1 \geq 0 .
\end{gathered}
$$

The fourth type is a state transition due to region transition of an on-going call from the normal region to the handoff region. In this situation, the call adjusts its data rate according to the status of the target BS. Let $\gamma_{k}$ be the probability that a BS has $k$ remaining resource. Because it is assumed that the state of the neighboring BS is similar to the state of the serving BS in steady state, $\gamma_{k}$ is defined as

$$
\gamma_{k}=\left.\sum_{s=0}^{s_{\max }} p(s)\right|_{R_{\mathrm{n}}(s)+R_{\mathrm{h}}(s)=(C-k)} .
$$

Let $r_{\mathrm{ho}}\left(x_{2}, x_{1}\right)$ be the flow component from state $x_{1}$ to state $x_{2}$ due to region transition of an on-going call from the normal region to the handoff region. If we define the state $x_{k}$ as

$\left\{\begin{array}{ccc}c_{1}(s) & \cdots & \cdots \\ c_{1}^{\mathrm{hi}}(s) & & \cdots \\ c_{1}^{\mathrm{ho}}(s) & \cdots & \left(c_{n}^{\mathrm{ho}}(s)+1\right)\end{array}\right.$

$$
\left.\begin{array}{ccc}
\left(c_{k}(s)-1\right) & \cdots & c_{N}(s) \\
\cdots & & c_{N}^{\text {hi }}(s) \\
\cdots & \cdots & c_{N}^{\text {ho }}(s)
\end{array}\right\}
$$

where $(1 \leq k \leq N, 1 \leq n \leq k)$

then, $r_{\mathrm{ho}}\left(x_{k}, s\right)$ is expressed as

$$
\begin{aligned}
& r_{\mathrm{ho}}\left(x_{k}, s\right) \\
& \quad= \begin{cases}\mu_{\mathrm{th}} \cdot c_{k}(s) \cdot \sum_{i=k}^{C} \gamma_{i}, & \text { for } n=k, c_{k}(s)-1 \geq 0 \\
\mu_{\mathrm{th}} \cdot c_{k}(s) \cdot \gamma_{n}, & \text { for } 1 \leq n<k, c_{k}(s)-1 \geq 0 . \\
0, & \text { otherwise }\end{cases}
\end{aligned}
$$

The fifth type is a state transition due to handoff-call arrival. Let $r_{\mathrm{hi}}\left(x_{2}, x_{1}\right)$ be the flow component from state $x_{1}$ to state $x_{2}$ due to handoff-call arrival. Also, let $\beta_{k}$ be the probability that a handoff call that occupies $k$ resource arrives. It means the ratio of the number of calls that occupies $k$ resource among calls arriving in the handoff region. $k$ has the value ranging from 1 to $N$. Then, $\beta_{k}$ is derived from

$$
\beta_{k}=\frac{\sum_{s=0}^{s_{\max }} \mu_{\mathrm{th}} \cdot c_{k}(s) \cdot p(s)}{\sum_{i=1}^{N} \sum_{s=0}^{s_{\max }} \mu_{\mathrm{th}} \cdot c_{i}(s) \cdot p(s)}, \quad \text { for } 1 \leq k \leq N
$$

where $P_{\mathrm{B}}$ is the blocking probability, $p(s)$ is the steady-state probability of the state $s$, and $\mu_{\mathrm{th}}$ is the probability that a call in the normal region moves to the handoff region. In this equation, the numerator indicates the number of calls occupying $k$ resource, which moves from the normal region to the handoff region, and the denominator expresses the total number of calls that are moved from the normal region to the handoff region.
Also, the handoff-call-arrival rate $\lambda_{\mathrm{h}}$ can be derived as follows:

$$
\lambda_{\mathrm{h}}=\sum_{s=0}^{s_{\max }} \sum_{i=1}^{N} \mu_{\mathrm{th}} \cdot c_{i}(s) \cdot p(s)
$$

If we define the state $x_{\text {hi }}$ as

$\left\{\begin{array}{ccccc}c_{1}(s) & & \cdots & & c_{N}(s) \\ c_{1}^{\mathrm{hi}}(s) & \cdots & \left(c_{k}^{\mathrm{hi}}(s)+1\right) & \cdots & c_{N}^{\mathrm{hi}}(s) \\ c_{1}^{\mathrm{ho}}(s) & & \cdots & & c_{N}^{\text {ho }}(s)\end{array}\right\}$

where $(1 \leq k \leq N)$

then $r_{\mathrm{hi}}\left(x_{\mathrm{hi}}, s\right)$ is expressed as

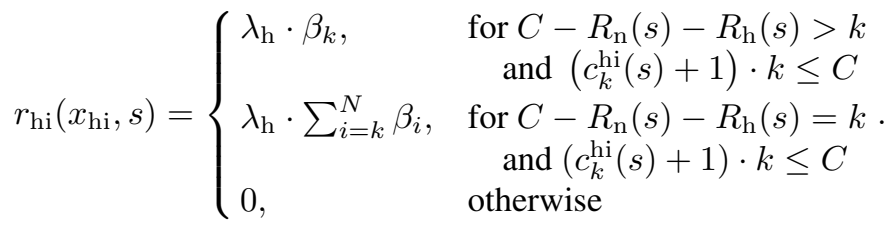

The sixth type is a state transition due to handoff out from the current cell. Let $r_{\mathrm{o}}\left(x_{2}, x_{1}\right)$ be the flow component from state $x_{1}$ to state $x_{2}$ due to handoff out from the current cell. If we define the state $x_{\mathrm{o}}$ as

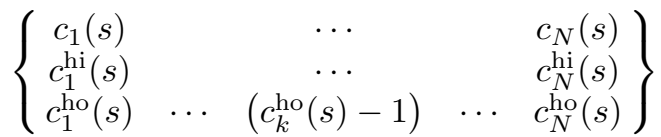

$$
\begin{aligned}
& \text { where }(1 \leq k \leq N)
\end{aligned}
$$

then $r_{\mathrm{o}}\left(x_{\mathrm{o}}, s\right)$ is expressed as

$$
r_{\mathrm{o}}\left(x_{\mathrm{o}}, s\right)=\mu_{\mathrm{tn}} \cdot c_{k}^{\mathrm{ho}}(s), \quad \text { for } c_{k}^{\mathrm{ho}}(s)-1 \geq 0 .
$$

The final type is a state transition due to region transition of a handoff-in call from the handoff region to the normal region of the serving cell. Let $\chi_{a}^{b}$ be the probability that a call that moves from the handoff region to the normal region of the serving cell occupies $a$ resource currently, but the call belongs to a service type that needs $b$ resource. Then, $\chi_{a}^{b}$ can be approximately derived as

$$
\chi_{a}^{b} \approx\left\{\begin{array}{cl}
\frac{\sum_{s=0}^{s_{\max }} \mu_{\mathrm{tn}} \cdot c_{a}^{\mathrm{hi}}(s) \cdot p(s)}{\sum_{i=1}^{N} \sum_{s=0}^{s_{\max }} \mu_{\mathrm{tn}} \cdot c_{i}^{\mathrm{hi}}(s) \cdot p(s)} & \\
\frac{\sum_{b} \cdot \gamma_{a}}{\sum_{j=(a+1)}^{N} \alpha_{j} \cdot \gamma_{a}+\alpha_{a} \cdot \sum_{k=a}^{C} \gamma_{k}}, & \text { for } a=b \\
\frac{\sum_{s=0}^{s_{\max }} \mu_{\mathrm{tn}} \cdot c_{a}^{\mathrm{hi}}(s) \cdot p(s)}{\sum_{i=1}^{N} \sum_{s=0}^{s_{\max }} \mu_{\mathrm{tn}} \cdot c_{i}^{\mathrm{hi}}(s) \cdot p(s)} & \\
\frac{\alpha_{a} \cdot \sum_{k=a}^{C} \gamma_{k}}{\sum_{j=(a+1)}^{N} \alpha_{j} \cdot \gamma_{a}+\alpha_{a} \cdot \sum_{k=a}^{C} \gamma_{k}}, & \text { for } a<b
\end{array}\right.
$$

The first fraction of (15) indicates the probability that a handoffin call that moves from the handoff region to the normal region of the serving cell consumes $a$ resource. The second fraction expresses the probability that a call that occupies $a$ resource serves service type $b$. Let $r_{i}\left(x_{2}, x_{1}\right)$ be the flow component from state $x_{1}$ to state $x_{2}$ due to region transition of a handoff-in 
call from the handoff region to the normal region. If we define the state $x_{k}$ as

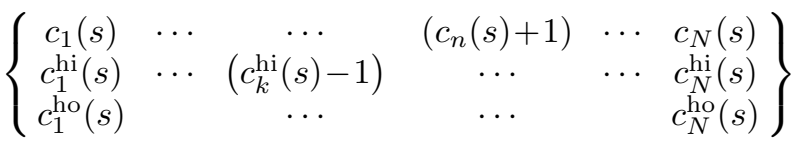

where $(1 \leq k \leq n \leq N)$

then, $r_{i}\left(x_{k}, s\right)$ is expressed as (16), shown at the bottom of the page, where $c_{k}^{\text {hi }}(s)-1 \geq 0$ and $\left(c_{n}(s)+1\right) \cdot n \leq C$. When a handoff-in call from neighboring cells is moving from the handoff region to the normal region, the call renegotiates its data rate according to its service type.

There are five sets of state-transition rate, which are symbolized by character from $\mathrm{A}_{k}$ to $\mathrm{E}_{k}$. $\mathrm{A}_{k}$ indicates the state transition when a new call that requires $k$ resource arrives in the normal region or an on-going call occupying $k$ resource in the normal region is completed. $\mathrm{B}_{k}$ indicates the state transition when a handoff-in call is moved from the handoff region to the normal region. $C_{k}$ indicates the state transition when an on-going call is moved from the normal region to the handoff region. $\mathrm{D}_{k}$ indicates the state transition when a new call that requires $k$ resource arrives in the handoff region or a handoff call occupying $k$ resource arrives or a handoff-in call occupying $k$ resource is completed. $\mathrm{E}_{k}$ indicates the state transition when a new call that requires $k$ resource arrives in the handoff region or a handoff-in call occupying $k$ resource is completed or a call occupying $k$ resource in the handoff region is moved out from current cell. These sets are shown in Fig. 3. There are some conditions that make each set operate. These conditions are derived from the state-transition types that organize the set.

\section{Performance Measures}

We define the steady-state probability of the state " $s$ " to be $p(s)$. Using the condition that the sum of the steady-state probability is equal to one, we can solve flow equilibrium equations and find steady-state probabilities. We also use a computer-programmed iterative approach to obtain the stationary state probabilities. We evaluate the performance of the DASH scheme in view of the blocking probability, the handoff failure probability, and the carried traffic.

A new call is blocked when there is no sufficient resource. If there remains $k$ available resources in a cell in case that a new call requests $k+1$ or more resources, the new call will be blocked. Therefore, the subset of states in which a blocking event can occur is described as

$$
B_{i}=\left\{s: R_{\mathrm{n}}(s)+R_{\mathrm{h}}(s)=C-i+1,1 \leq i \leq N\right\} .
$$

Ak:

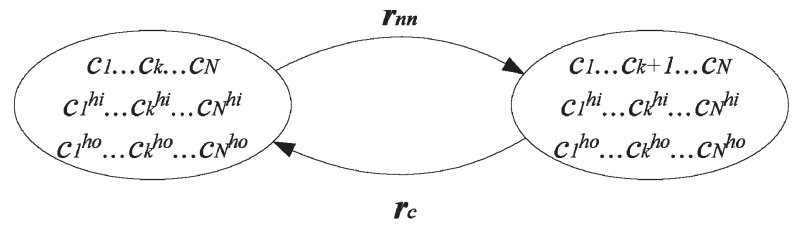

Bk:

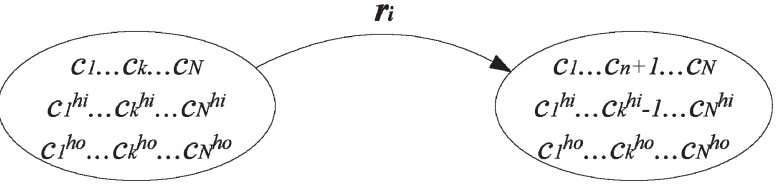

Ck:
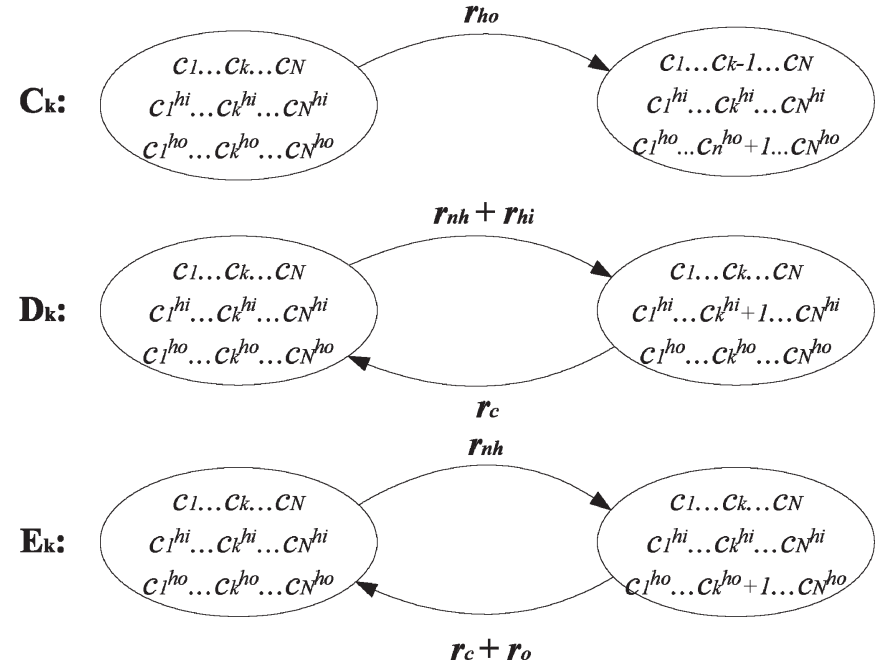

Fig. 3. Sets of state-transition rate.

Because a blocking event occurs when the cell is in a state $s$, which belongs to $B_{i}$, the blocking probability is calculated as

$$
P_{\mathrm{B}}=\sum_{i=1}^{N} \sum_{s \in B_{i}} p(s) \cdot \sum_{j=i}^{N} \alpha_{j} .
$$

There are two cases when a handoff event fails. The first case is generated when a handoff call is dropped due to the shortage of remaining resource in the target cell. The second case happens when a new call originated in the handoff region gets a resource from only one cell and this call is dropped when it arrives at the boundary of its current cell. The handoff failure probability due to the first case is described as

$$
P_{\mathrm{H}_{1}}=\left.\sum_{s=0}^{s_{\text {max }}} p(s)\right|_{R_{\mathrm{n}}(s)+R_{\mathrm{h}}(s)=C} .
$$

The second case happens when a new call originated in the handoff region communicates with only the current cell but it moves to the other cell. Because there is no available resource in

$$
r_{i}\left(x_{k}, s\right)= \begin{cases}\mu_{\mathrm{tn}} \cdot c_{k}^{\mathrm{hi}}(s) \cdot\left(\left.\sum_{i=k}^{N} \chi_{k}^{i}\right|_{R_{\mathrm{n}}(s)+R_{\mathrm{h}}(s)=C}+\left.\chi_{k}^{n}\right|_{R_{\mathrm{n}}(s)+R_{\mathrm{h}}(s)<C}\right), & \text { for } k=n \\ \mu_{\mathrm{tn}} \cdot c_{k}^{\mathrm{hi}}(s) \cdot\left(\left.\sum_{i=n}^{N} \chi_{k}^{i}\right|_{R_{\mathrm{n}}(s)+R_{\mathrm{h}}(s)=C-n+k}+\left.\chi_{k}^{n}\right|_{R_{\mathrm{n}}(s)+R_{\mathrm{h}}(s)<C-n+k}\right), & \text { for } k<n \\ 0, & \text { otherwise }\end{cases}
$$


the target cell, this call is dropped. Therefore, the total handoff failure probability is obtained by

$$
\begin{aligned}
P_{\mathrm{H}}=\frac{\lambda_{\mathrm{h}}}{\lambda_{\mathrm{h}}+0.5 \cdot \lambda_{\mathrm{nh}}} & \cdot \sum_{i=1}^{N} P_{\mathrm{H}_{1}} \\
& +\frac{0.5 \cdot \lambda_{\mathrm{nh}}}{\lambda_{\mathrm{h}}+0.5 \cdot \lambda_{\mathrm{nh}}} \cdot P_{\mathrm{B}} \cdot\left(1-P_{\mathrm{B}}\right) .
\end{aligned}
$$

In this equation, the front part indicates the first case and the latter part indicates the second case.

The carried traffic indicates the amount of transmitted data in steady state and shows the efficiency of resource usage. The carried traffic $T$ is defined as

$$
T=\sum_{s=0}^{s_{\max }}\left(R_{\mathrm{n}}(s)+R_{\mathrm{h}}(s)\right) \cdot p(s) .
$$

\section{NUMERICAL RESUltS}

We analyze and simulate the performance of the DASH scheme. In our simulation, we consider circular cells whose BSs are located along the streets. This cell structure reflects road-vehicle ITS. Our simulation model consists of 37 cells and the radius of a cell is $100 \mathrm{~m}$. The ratio of the handoff region in a cell is 0.3. MSs are uniformly distributed in each cell. The call-arrival rate and call-duration time of the simulation is the same as those of the analysis. Each MS moves in just two directions. No turning back and no changing direction are allowed. We perform ten iterations for this simulation and average the results. The simulation time of each iteration is $1000000 \mathrm{~s}$.

The traffic model that we consider in this numerical example is real-time streaming traffic such as $\mathrm{VoD}$ and videophone. We assume that a call has infinite amount of data in the simulation, and $T_{\mathrm{C}}$ has infinite value in the analysis. Because real-time streaming services are mainly served for vehicle users, we assume that the speed of an MS is $30 \mathrm{~km} / \mathrm{h}$ in the simulation and $T_{\mathrm{dg}}$ is $24 \mathrm{~s}$ in the analysis. We investigate numerical example in the case that the ratio of the handoff region $f$ is 0.3 , the total allowable resource of a cell $C$ is 10 , and the number of service type $N$ is 3 . In the numerical example, we change the probability that a new call belongs to a service type that requires $k$ resource $\alpha_{k}$. As we assumed that $N=3$, there are three kinds of services and $\alpha_{1}+\alpha_{2}+\alpha_{3}=1$. We evaluate the performance of the DASH scheme with respect to the blocking probability, the handoff failure probability, and the carried traffic. To show the effect of the DASH scheme on the system performance, numerical results are compared with basic soft-handoff scheme. The basic soft-handoff scheme that is considered in this paper is described in the cdma2000 standards [16].

Figs. 4-6 illustrate the blocking probability, the handoff failure probability, and the carried traffic of the basic soft-handoff scheme and the DASH scheme, respectively. The results show close agreement between the simulation and analytical models. These figures show that the blocking probability of the DASH scheme is slightly higher than that of the basic soft-handoff

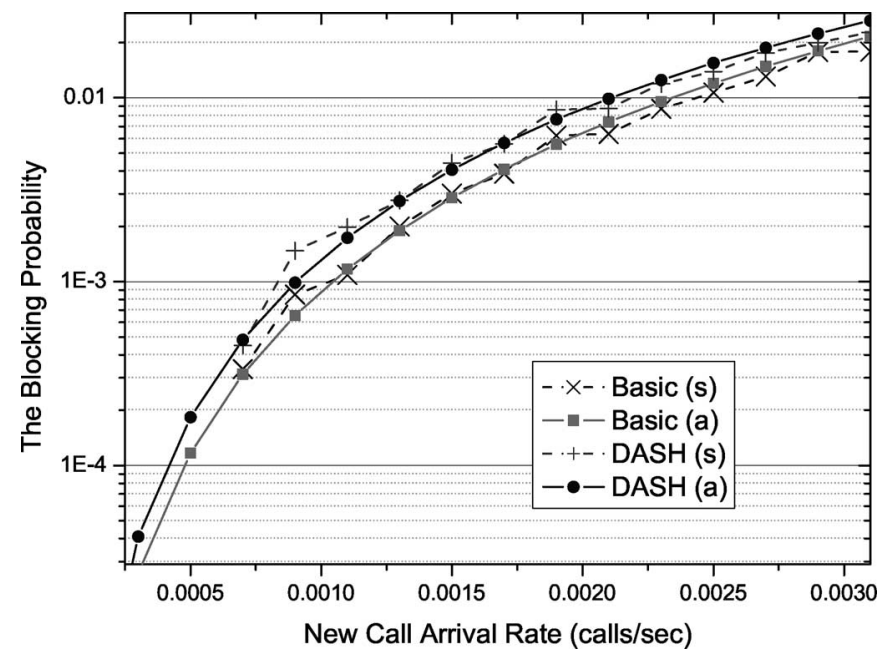

Fig. 4. Comparison in view of the blocking probability; $f=0.3, C=10$, $N=3, \alpha_{1}=0.2, \alpha_{2}=0.2, \alpha_{3}=0.6$; (s) simulation, (a) analysis.

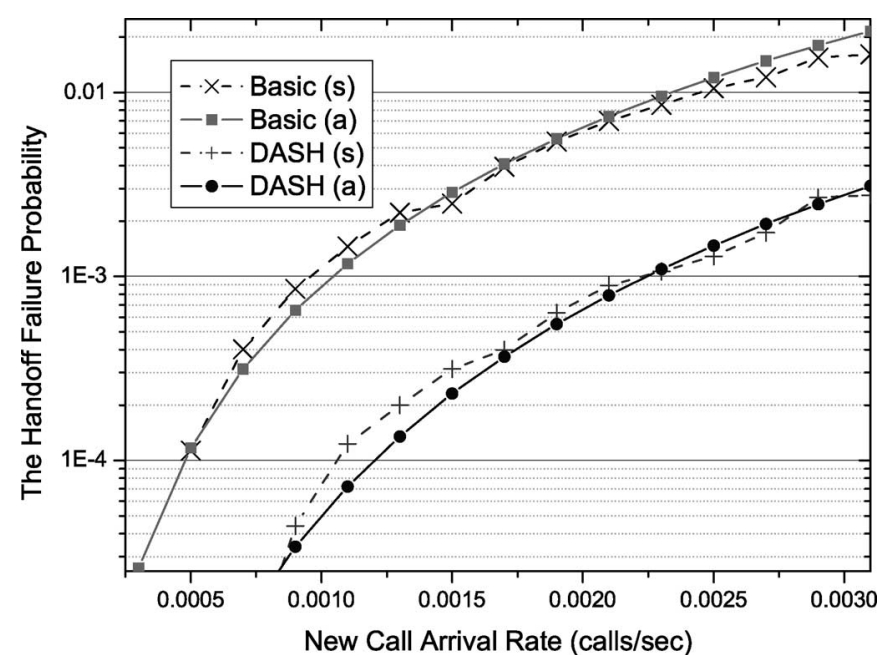

Fig. 5. Comparison in view of the handoff failure probability; $f=0.3$, $C=10, \quad N=3, \quad \alpha_{1}=0.2, \quad \alpha_{2}=0.2, \quad \alpha_{3}=0.6 ; \quad$ (s) simulation, (a) analysis.

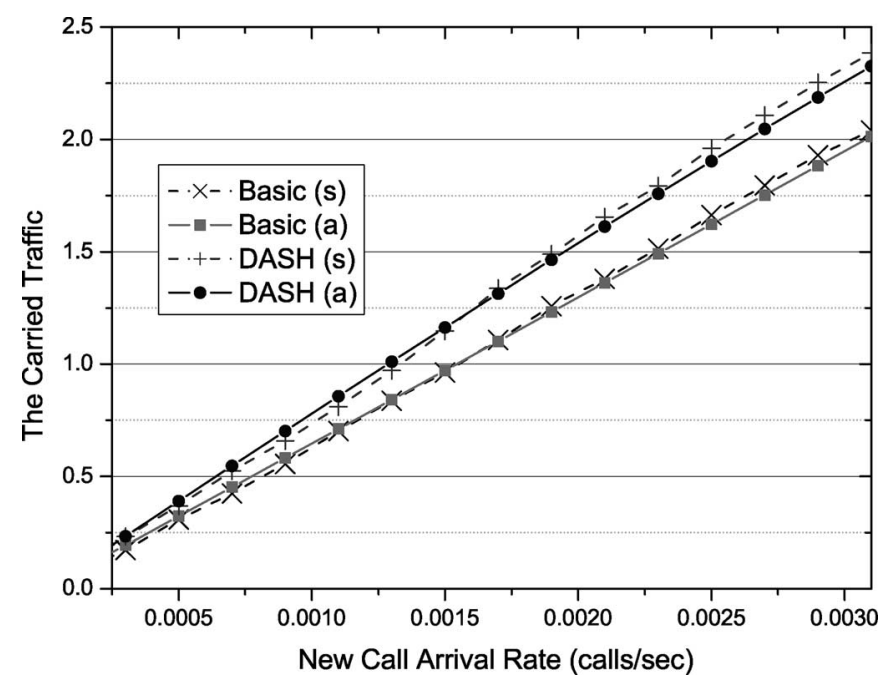

Fig. 6. Comparison in view of the carried traffic; $f=0.3, C=10, N=3$, $\alpha_{1}=0.2, \alpha_{2}=0.2, \alpha_{3}=0.6$; (s) simulation, (a) analysis. 


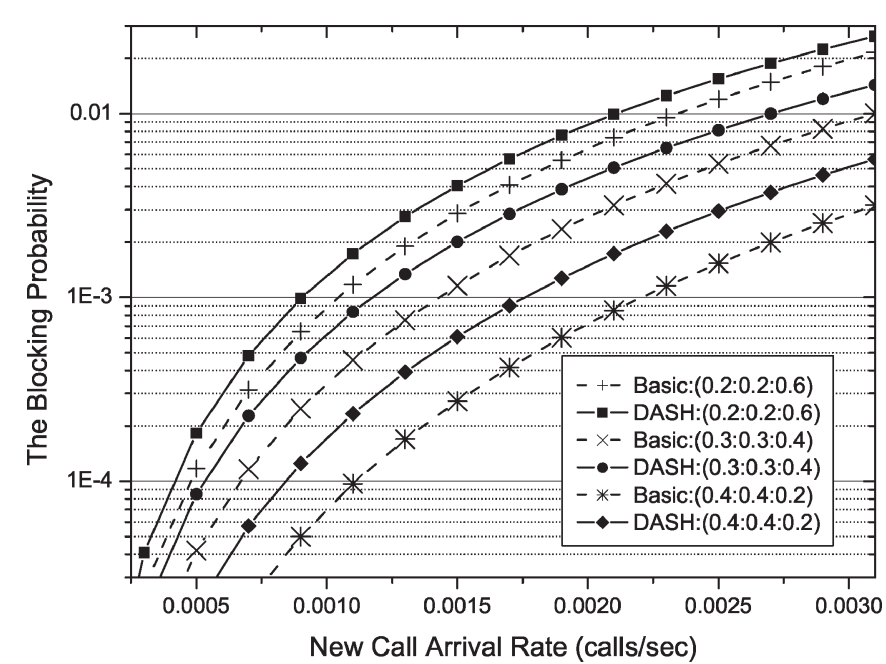

Fig. 7. Blocking probability with changing the service-type constitution; $f=0.3, C=10, N=3$.

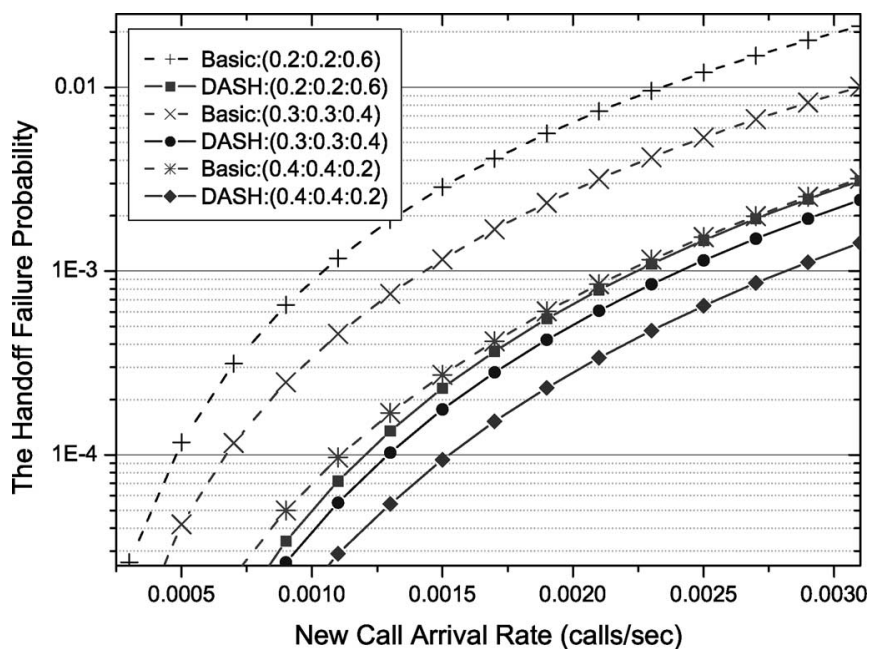

Fig. 8. Handoff failure probability with changing the service-type constitution; $f=0.3, C=10, N=3$.

scheme. However, the handoff failure probability of the DASH scheme is much lower than that of the basic softhandoff scheme. The difference in the handoff failure probability is about six times of the difference in the blocking probability. The low handoff failure probability of the DASH scheme occurs because handoff-in calls with a high data rate can decrease their data rate when there is a shortage of resource in the target cell. Also, we can see that the carried traffic of the DASH scheme is bigger than that of the basic soft-handoff scheme. From these results, we can see that the DASH scheme makes seamless service possible and permits good performance in view of throughput.

Figs. 7-9 show performances when the service-type constitution $\left(\alpha_{1}: \alpha_{2}: \alpha_{3}\right)$ is changed. As the ratio of $\alpha_{3}$ increases, all performance measures such as the blocking probability, the handoff failure probability, and the carried traffic increase. The difference of the handoff failure probability between the DASH scheme and the basic soft-handoff scheme becomes bigger, as the ratio of service type that requires many resource increases. However, the difference of the blocking probability between the

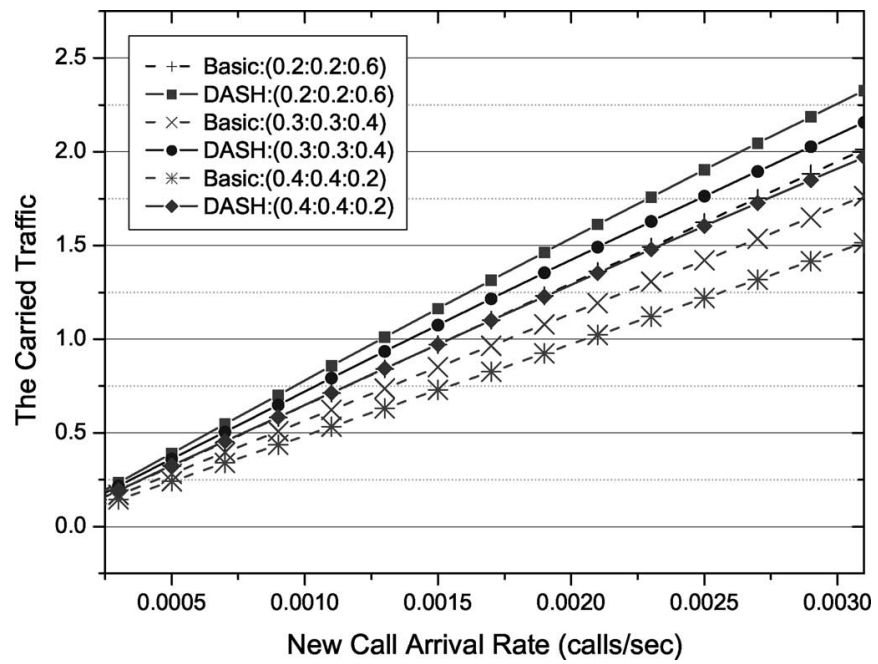

Fig. 9. Carried traffic with changing the service-type constitution; $f=0.3$, $C=10, N=3$.

DASH scheme and basic soft-handoff scheme maintains a similar value, as the ratio of service type that requires many resource increases. These results show that the DASH scheme is suitable for users with a high data rate rather than a low data rate.

In this paper, we define that the QoS of a service is guaranteed when the service is provided with the data rate that is initially defined by service type. Additionally, the notation "seamless service" indicates the ability that a call provides a service without any termination. Assume that the data rate for an MS is adjusted according to the proposed scheme. If the adjusted data rate is lower than the previous data rate, the serving BS should diminish the transmission power to the MS of interest according to the adjusted data rate. Then, the BS sends messages to the source of streaming service for informing the change of the supported data rate, and the streaming source changes the video encoding rate to support seamless service. It causes the degradation of video/image quality or QoS. Using the similar process, the QoS is upgraded when the adjusted data rate is higher than the previous data rate. Basic soft handoff can guarantee QoS. However, the high handoff failure probability shows that it is vulnerable to support seamless service. On the other side, the DASH scheme supports seamless call handling rather than the QoS guarantee. The DASH scheme can utilize resource more efficiently but has a complexity problem.

From the numerical results, the application of each scheme can be divided as follows: The DASH scheme is suitable for users moving with high mobility because it gives relative good maintenance of calls. Under the environment that requires low mobility, the basic soft-handoff scheme can be a good candidate. From a different standpoint, the DASH scheme is suitable under the environment that requires a high data rate. It is adequate that users with low data rate are supported by the basic soft-handoff scheme. These recommended applications are summarized in Table I.

\section{CONCLUSION}

A soft-handoff scheme for real-time streaming services in CDMA-based fITS was proposed. The proposed DASH scheme 
TABLE I

RECOMMENDED APPLICATIONS OF THE DASH AND BASIC SOFT-HANDOFF SCHEMES

\begin{tabular}{l||c|c}
\hline \hline & Mobility & Data Rate \\
\hline \hline DASH & High & High \\
\hline Basic & Low & Low \\
\hline \hline
\end{tabular}

adjusts the data rate of a call when it starts and finishes soft handoff. The DASH scheme can give relative good maintenance of calls regardless of the shortage of resource. Also, it can maximize the resource efficiency. Using analytic and simulation methods, we evaluated the performance of the DASH scheme. Performances were evaluated with respect to the blocking probability, the handoff failure probability, and the carried traffic. Numerical results were compared with the basic soft-handoff scheme. Comparisons revealed that the DASH scheme makes seamless service possible and permits good throughput. From the numerical results, we concluded that the DASH scheme is adequate for the environment that has many fast-moving users with high data rate. These results can present designing points to develop an efficient resource-management strategy for CDMA-based ITS systems that support real-time streaming services.

\section{REFERENCES}

[1] H. Harada, K. Sato, and M. Fujise, "A radio-on-fiber based millimeterwave road-vehicle communication system by a code division multiplexing radio transmission scheme," IEEE Trans. Intell. Transp. Syst., vol. 2, no. 4, pp. 165-179, Dec. 2001.

[2] Y. Zhao, "Mobile phone location determination and its impact on intelligent transportation systems," IEEE Trans. Intell. Transp. Syst., vol. 1, no. 1, pp. 55-64, Mar. 2000

[3] A. J. Viterbi, A. M. Viterbi, K. S. Gilhousen, and E. Zehavi, "Soft handoff extends CDMA cell coverage and increase reverse link capacity," IEEE J. Sel. Areas Commun., vol. 12, no. 8, pp. 1281-1288, Oct. 1994.

[4] C.-C. Lee and R. Steele, "Effect of soft and softer handoffs on CDMA system capacity," IEEE Trans. Veh. Technol., vol. 47, no. 3, pp. 830-841, Aug. 1998.

[5] S. L. Su, J. Y. Chen, and J. H. Huang, "Performance analysis of soft handoff in CDMA cellular networks," IEEE J. Sel. Areas Commun., vol. 14, no. 9, pp. 1762-1769, Dec. 1996

[6] R. P. Narrainen and F. Takawira, "Performance analysis of soft handoff in CDMA cellular networks," IEEE Trans. Veh. Technol., vol. 50, no. 6, pp. 1507-1517, Dec. 2001.

[7] J. Wu and R. Kohno, "A wireless multimedia CDMA system based on transmission power control," IEEE J. Sel. Areas Commun., vol. 14, no. 4, pp. 683-691, May 1996.
[8] M. Soroushnejad and E. Geraniotis, "Multiple-access strategies for an integrated voice/data CDMA packet radio network," IEEE Trans. Commun., vol. 43, no. 2-4, pp. 934-945, Feb.-Apr. 1995.

[9] M. L. Honig and J. B. Kim, "Allocation of DS-CDMA parameters to achieve multiple rates and qualities of service," in Proc. GLOBECOM, 1996, pp. 1974-1978.

[10] A. Sampath and J. M. Holtzman, "Access control of data in integrated voice/data CDMA systems: Benefits and tradeoffs," IEEE J. Sel. Areas Commun., vol. 15, no. 8, pp. 1511-1526, Oct. 1997.

[11] T. Liu and J. A. Silvester, "Joint admission/congestion control for wireless CDMA systems supporting integrated services," IEEE J. Sel. Areas Commun., vol. 16, no. 6, pp. 845-857, Aug. 1998.

[12] N. Dimitriou and R. Tafazolli, "Quality of services for multimedia CDMA," IEEE Commun. Mag., vol. 38, no. 7, pp. 88-94, Jul. 2000.

[13] R. Fantacci and S. Nannicini, "Multiple access protocol for integration of variable bit rate multimedia traffic in UMTS/IMT-2000 based on wideband CDMA," IEEE J. Sel. Areas Commun., vol. 18, no. 8, pp. 14411454, Aug. 2000

[14] I. F. Akyildiz et al., "Medium access control protocols for multimedia traffic in wireless networks," IEEE Netw., vol. 13, no. 4, pp. 39-47, Jul./Aug. 1999.

[15] Y. Chung and D. H. Cho, "Performance evaluation of soft handoff for multimedia services in intelligent transportation systems based on CDMA," IEEE Trans. Intell. Transp. Syst., vol. 4, no. 4, pp. 189-197, Dec. 2003.

[16] “3GPP2 C.S0005-A," Upper Layer (Layer 3) Signaling Standard for cdma2000 Spread Spectrum Systems, Jun. 2000.

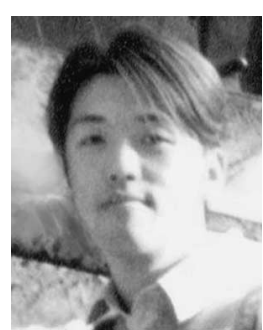

Young-uk Chung received the B.S., M.S., and Ph.D. degrees in electrical engineering and computer science from the Korea Advanced Institute of Science and Technology (KAIST), Daejeon, Korea, in 1997, 1999, and 2003, respectively.

From 2003 to 2005 , he was a manager with Hynix Semiconductor Inc., Ltd. Since 2005, he has been with Kwangwoon University, Seoul, Korea, where he is an Assistant Professor in the Department of Electronic Engineering. His research interests include wired/wireless communication network, and wireless multimedia systems.

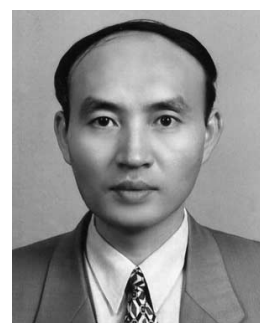

Dong-Ho Cho (M'85-SM'00) received the B.S degree in electrical engineering from Seoul National University, Seoul, Korea, in 1979 and the M.S. and Ph.D. degrees in electrical engineering from the Korea Advanced Institute of Science and Technology (KAIST), Daejeon, Korea, in 1981 and 1985, respectively.

From 1987 to 1997 , he was a Professor in the Department of Computer Engineering at Kyunghee University. Since 1998, he has been with KAIST, where he is a Professor in the Department of Electrical Engineering and Computer Science. His research interests include wired/wireless communication network, protocol, and services. 